\title{
Potencial de Uso de Biogás en Colombia
}

\section{Potential Uses of Biogas in Colombia}

\author{
Melvin Acosta Pabuena', Jorgelina Pasqualino² \\ ${ }^{1}$ Estudiante, Ingeniería Ambiental, Semillero Ingenio Ambiental (IGA), Fundación Universitaria Tecnológico Comfenalco \\ (FUTCO), Sede A, Barrio España, Cra. 44D No. 30A - 91, Cartagena, Colombia. \\ melvinacostap@gmail.com \\ ${ }^{2}$ Docente investigador, Ingeniería Ambiental, Grupo de Investigación Ambiental (GIA), Fundación Universitaria Tecnológico \\ Comfenalco (FUTCO), Sede A, Barrio España, Cra. 44D No. 30A - 91, Cartagena, Colombia. \\ jpasqualino@tecnocomfenalco.edu.co
}

Recibido: 20Ago2014 - Revisado: 24Oct2014

Aceptado: 24Nov2014 - Publicado: 10Dic2014

\begin{abstract}
Resumen: En Colombia, aunque en promedio, el 93\% de los residuos sólidos se disponen en rellenos sanitarios adecuados, en el departamento de Bolívar, solo el $45 \%$ de la población recibe servicio de recolección, mientras que unas 150 ton/día se vierten de forma descontrolada en botaderos satélite y cuerpos de agua. En las zonas rurales, la cobertura alcanza apenas el 0,5\%. La fracción orgánica de los residuos urbanos alcanza hasta los 2/3. La digestión anaerobia de estos residuos para obtener biogás es ampliamente empleada en países de Europa y Asia. Sin embargo, en Latinoamérica, la producción de biogás tanto doméstica como industrial es escasa. En Colombia, se encuentran algunos digestores de tipo industrial en rellenos sanitarios y plantas procesadoras de aceite de palma y, aunque algunas de estas plantas utilizan el biogás con fines energéticos, en muchos casos el gas es quemado en antorcha como medio de reducción de emisiones de gases de efecto invernadero. En las zonas rurales de Colombia, se han implementado pequeños proyectos por iniciativa de los agricultores o de agencias de cooperación. En este artículo se presentarán los proyectos actuales de producción de biogás en Colombia y el potencial existente.
\end{abstract}

Palabras claves: Digestión anaerobia, residuos orgánicos, biogás, energías renovables.

\begin{abstract}
Although in Colombia about 93\% of the solid wastes is adequately disposed in sanitary landfills, the department of Bolivar reports only $45 \%$ of the population receiving waste collection services, while about 150 tons/day of solid wastes are disposed in uncontrolled manner, mostly around water courses or abandoned lands. In the rural sector, the waste collection service coverage reaches just $0,5 \%$. The organic fraction of urban solid wastes in Colombia reaches $2 / 3$ of the total. Anaerobic digestion of organic wastes for biogas production is widely used in European and Asian countries. However, Latinamerican biogas production, both at domestic or industrial scale, is scarce. In Colombia, one can find some industrial biodigesters in sanitary landfills and palm oil mills and, while some of them use biogas as an energy source, most of them burn it in torches as a means for greenhouse gases emissions reduction. Rural areas in Colombia have some small projects implemented with farmers' initiative or with the support of cooperation agencies. In this paper, the state of the art of current biogas projects in Colombia is presented as well as their potential.
\end{abstract}

Key words: Anaerobic digestion, organic wastes, biogas, renewable energies.

\section{INTRODUCCIÓN}

La digestión anaeróbica de la materia orgánica se produce mediante las siguientes etapas: desintegración/hidrólisis; acidogénesis (formación de ácidos); acetogénesis (formación de acetona) y metanogénesis (formación de metano) (Flotats, 2010), obteniendo al final un producto o biogás con las siguientes características: $\mathrm{CH}_{4}(50-75 \%), \mathrm{CO}_{2}(25-50 \%), \mathrm{N}_{2}$ $(0-5 \%), \mathrm{H}_{2} \mathrm{O}(1-5 \%)$, y trazas de $\mathrm{H}_{2} \mathrm{~S}$ y $\mathrm{NH}_{3}$, siendo mayor el contenido energético cuanto mayor es la concentración de $\mathrm{CH}_{4}$ (Surendra, 2014). Tanto la composición del biogás como el rendimiento del proceso (generalmente expresado como $\mathrm{m}^{3}$ de biogás/tonelada de materia seca), dependen de la materia prima procesada y de las condiciones de operación, tales como temperatura, $\mathrm{pH}$, diseño del biodigestor, agregado de inóculo, carga orgánica alimentada, tiempo de retención, entre otros (Ferrer 2011). Entre las materias primas más utilizadas para la producción de biogás encontramos el estiércol animal, los residuos agrícolas, los residuos orgánicos domésticos y los lodos de aguas residuales (Surendra, 2014), pudiendo usar la codigestión de diferentes sustratos para incrementar la productividad y calidad del gas obtenido, aprovechando los efectos sinérgicos. Como sub-producto del proceso, se obtiene un lodo que puede ser utilizado 
como fertilizante, debido a su elevada carga nutricional, ya que contiene aquellos nutrientes contenidos en la materia prima (Cendales, 2011).

Basándonos en lo anterior, el objetivo de este artículo es realizar una revisión de la situación actual de producción de biogás en Colombia y el potencial existente a partir del uso de residuos urbanos. Para ello, se incluye una descripción general de la situación de la tecnología de biogás en el mundo, específicamente en Colombia, y se evalúa el potencial de los residuos orgánicos urbanos como materia prima. Finalmente, se presenta un modelo de prototipo propuesto para aplicaciones domésticas.

\section{ANTECEDENTES}

La situación energética mundial se caracteriza por la desigualdad tanto de la oferta como de la demanda, siendo, además, la energía el elemento limitante que frena o retrasa el desarrollo económico de una región. La revolución energética en el futuro cercano debe estar marcada por dos grandes metas: suministro descentralizado (especialmente como abastecimiento a las comunidades vulnerables) y sustitución de las fuentes de energía basadas en el carbono fósil por energías que no incrementen la concentración de $\mathrm{CO}_{2}$ en la atmósfera. Estos cambios deben ir acompañados de la implementación masiva de Fuentes No Convencionales de Energía (FNCE) para poder concretarse.

El uso de FNCE en Colombia en el periodo 1990-2009 representó el 21,38\% de la oferta total (1.534478 T J/año en promedio), con la siguiente distribución: 9,46\% hidroeléctrica, 7,10\% madera, 4,59\% pulpa de caña de azúcar, 0,013\% energía eólica, 0,08\% biodiesel y 0,14\% bioetanol (IAvH, 2011). No se evidencia aporte de energía procedente de biogás; presentando, sin embargo, un elevado porcentaje de uso de la madera como fuente de energía, la cual es utilizada en Colombia principalmente como fuente de energía térmica (cocción, calefacción, etc.).

Las Zonas No-Interconectadas (ZNI) en Colombia corresponden al $66 \%$ del territorio nacional, con el $4 \%$ de la población principalmente en zonas rurales o aisladas. Alrededor del $10 \%$ de la energía consumida en estas zonas correspondea FNCE (comolas energías fotovoltaicas, biomasa y minihidroeléctrica), mientras que el resto de consumo es suplido con generadores diesel para producción de energía eléctrica (MME, 2010). Debido a su situación geográfica, dificultad de acceso y el precio de los combustibles, los usuarios de generadores diesel suelen pagar el doble de precio por KW/h en relación a las zonas conectadas a la red nacional, a pesar de disponer de un servicio de baja calidad con un número de horas reducido de prestación. La demanda de energía térmica en estas regiones es suplida en su mayor parte mediante el uso de madera. Sin embargo, estas prácticas causan contaminación del aire en el interior de las viviendas, debido principalmente a emisiones de partículas y gases procedentes de la combustión incompleta, lo que causa enfermedades respiratorias a los usuarios. Además, el uso extensivo de madera como fuente de energía tiene un impacto considerable en la vegetación local (Surendra, 2014; Subedi 2014). En Colombia, por ejemplo, las zonas de manglares y de bosque seco tropical de la costa Caribe han sido gravemente afectadas por el uso de la madera como material de construcción y como combustible, causando erosión del suelo, desertificación, degradación e incremento de las emisiones de gases de efecto invernadero.

\section{TECNOLOGÍA DE BIOGÁS}

\subsection{Aplicación del Biogás}

El biogás puede ser empleado en diferentes aplicaciones que cubren los usos a nivel doméstico, comunitario e industrial. A escala doméstica, se pueden emplear biodigestores de 2-16 $\mathrm{m}^{3}$ de capacidad, generalmente para aplicaciones de cocción, calefacción e iluminación, como autoabastecimiento en zonas rurales o aisladas en las cuales se generan cantidades considerables de residuos orgánicos (Surendra, 2014; Song, 2014; Raha, 2014; Walekhwa, 2014; Mwirigi, 2014). A escala comunitaria, pueden emplearse biodigestores de mayores capacidades como suministro de biogás para poblaciones compuestas por varias viviendas agrupadas, para edificios comunitarios como colegios o comedores, para suministro de servicios públicos como iluminación urbana, o para comercios como los restaurantes. Los biodigestores normalmente utilizados tanto a nivel doméstico como comunitario son de tipo manga tubular o semienterrados de domo flotante o fijo. Las características de cada uno de ellos los diferencian en función de los materiales de los que están construidos (cemento, plástico, acero inoxidable, etc.), su ubicación (enterrados o elevados), orientación (horizontales o verticales), y tipo de carga (continua o discontinua) (Guardado Chacón, 2007; Pinzón, 2004; Orskov, 2014). Los biodigestores de tipo industrial pueden ser utilizados como fuente de energía térmica, en plantas de ciclo combinado para cogeneración de calor y electricidad o como combustible de transporte, aunque algunas de estas aplicaciones requieren la purificación del biogás para eliminar los gases diferentes del metano. Este tipo de biodigestores pueden encontrarse en rellenos sanitarios, plantas de tratamiento de aguas residuales (Pasqualino, 2009), industrias procesadoras de alimentos, agroindustrias (tales como las plantas procesadoras de aceite de palma) (Arrieta, 2007; Conil, 2009), entre otras.

\subsection{Situación Mundial}

La tecnología de digestión anaerobia para obtención de biogás se ha utilizado ampliamente en países de Europa, especialmente en Alemania, donde la legislación promueve y apoya proyectos de producción de biogás, especialmente en el sector rural. En Asia, los países que han desarrollado 
Tabla 1. Proyectos de biogás reportados en Colombia

\begin{tabular}{|c|c|c|}
\hline $\begin{array}{l}\text { Proyecto nombre o } \\
\text { localización }\end{array}$ & Descripción & Referencias \\
\hline Palmeras del Llano & $\begin{array}{l}\text { Planta de extracción de aceite de palma. Biodigestor hormigón, } 750 \text { m³ de capacidad. } \\
\text { Aplicación biogás para la generación de electricidad. Instalado en } 1987 .\end{array}$ & Conil, 2009 \\
\hline Palmar Santa Elena & $\begin{array}{l}\text { Planta de extracción de aceite de palma. Biodigestor hormigón, } 500 \text { m³ de capacidad. } \\
\text { Aplicación biogás para la generación de electricidad. Instalado en } 1991 \text {. }\end{array}$ & Conil, 2009 \\
\hline Palmeiras & $\begin{array}{l}\text { Planta de extracción de aceite de palma. Carpa cubierta laguna, } 7.000 \mathrm{~m}^{3} \text { de } \\
\text { capacidad. Aplicación del biogás para la generación de electricidad. Instalado en } 1999 .\end{array}$ & Conil, 2009 \\
\hline Cali & $\begin{array}{l}\text { Proyecto MDL: Autogeneración de biogás como energía renovable en la Planta de } \\
\text { Tratamiento de Aguas Residuales de Cañaveralejo de EMCALI. }\end{array}$ & $\begin{array}{l}{ }^{*} \mathrm{MDL}, \mathrm{UNFCCC}, \\
2009\end{array}$ \\
\hline Cali & $\begin{array}{l}\text { Proyecto MDL: Recuperación y combustión de metano en el tratamiento de lodos } \\
\text { existente sistema de la Planta de Tratamiento de Aguas Residuales de Cañaveralejo de } \\
\text { EMCALI. }\end{array}$ & $\begin{array}{l}\text { *MDL, UNFCCC, } \\
2009\end{array}$ \\
\hline Pasto & $\begin{array}{l}\text { Proyecto MDL: Construcción, operación y mantenimiento de sistema de colección y } \\
\text { quema de biogás en el relleno sanitario Antanas. }\end{array}$ & $\begin{array}{l}\text { *MDL, UNFCCC, } \\
2009\end{array}$ \\
\hline $\begin{array}{l}\text { Puerto Leguizamo } \\
\text { (Putumayo) }\end{array}$ & Planta piloto de Biogás para generación de energía. & MME, 2010 \\
\hline $\begin{array}{l}\text { San Carlos de Guaroa } \\
\text { (Meta) }\end{array}$ & $\begin{array}{l}\text { Aceites Manuelita, } 2 \text { biodigestores } 19.000 \mathrm{~m}^{3} \text {, procesa } 300.000 \text { Ton/año de material, } \\
\text { aplicación en Planta de Biodiesel. }\end{array}$ & Biotec, 2013 \\
\hline $\begin{array}{l}\text { Sabana de Torres } \\
\text { (Santander) }\end{array}$ & $\begin{array}{l}\text { Oro Rojo, Grupo Indulpalma, biodigestor } 16.000 \text { m³, procesa } 150.000 \text { ton/año de } \\
\text { material, sin aplicación (tea). }\end{array}$ & Biotec, 2013 \\
\hline
\end{tabular}

*MDL: Mecanismo de Desarrollo Limpio, UNFCCC: United Nations Framework Convention on Climate Change

ampliamente esta tecnología son China e India, donde se ha implementado el uso de biodigestores en zonas rurales y pequeñas comunidades (Cendales, 2011; Ferrer, 2011; Song, 2014; Raha, 2014). En China, por ejemplo, se construyeron 5 millones de nuevas unidades en el año 2010, alcanzando un total de 40 millones de biodigestores. En India, se reportaron 4, millones de biodigestores en operación en 2011, con el apoyo del Programa Nacional para la gestión de Biogás y Fertilizantes (Mwuirigi, 2014). En África, se están desarrollando varios programas de fomento de esta tecnología, como los programas nacionales de biogás doméstico, entre ellos la Asociación Africana de Biogás, que ha promovido la construcción de unas 70.000 plantas de biogás en Ruanda, Tanzania, Kenia, Uganda, Etiopía, Camerún, Benín y Burkina Faso, con la colaboración económica de la Organización Holandesa de Desarrollo (Orskov, 2014; Mwirigi, 2014). En Latinoamérica, la tecnología de producción de biogás se ha desarrollado escasamente, debido a la falta de disponibilidad de la tecnología, los costos de inversión inicial y la fácil disponibilidad de madera como combustible en zonas rurales. Se han implementado pequeñas instalaciones en zonas rurales, en Colombia, Costa Rica, Perú y Bolivia, como iniciativa de los propios campesinos $y$, en algunos casos, con el apoyo de agencias de cooperación internacional (Cendales, 2011; Ferrer 2011; Pérez, 2014). Como ejemplo, se reporta el uso de digestores tubulares de plástico tanto en zonas tropicales como en zonas andinas, reportando buenos resultados (Ferrer, 2011).

\subsection{Situación en Colombia}

El uso de biodigestores domésticos en Colombia a escala rural se ha venido desarrollando lentamente, dado el escaso apoyo institucional que se ha dado a este tipo de proyectos. Sin embargo, la aplicación de esta tecnología en zonas andinas y algunas demostraciones a nivel doméstico han fomentado el crecimiento del sector en los últimos años. No se dispone de un registro completo de las pequeñas instalaciones disponibles a nivel rural, debido, principalmente, a que la mayor parte de las mismas son consecuencia de la iniciativa de los usuarios sin contar con un apoyo externo. A mayor escala, se reportan algunas experiencias, tal como se observa en la Tabla 1. Solo 3 proyectos se han reportado en Colombia dentro de los Mecanismos de Desarrollo Limpio; sin embargo, el auge de los cultivos de palma y la necesidad de tratamiento de los efluentes líquidos originados en las extractoras de aceite ha fomentado la implementación de proyectos en este sector.

\section{PotenCial en COlOMBiA}

En Colombia, en 2009 aproximadamente, el 93\% de los residuos sólidos fueron dispuestos de forma adecuada, la mayor parte de los mismos en rellenos sanitarios. El resto fueron dispuestos en cuerpos de agua, en botaderos a cielo abierto, fueron quemados o enterrados (DNP, 2011; IAvH, 


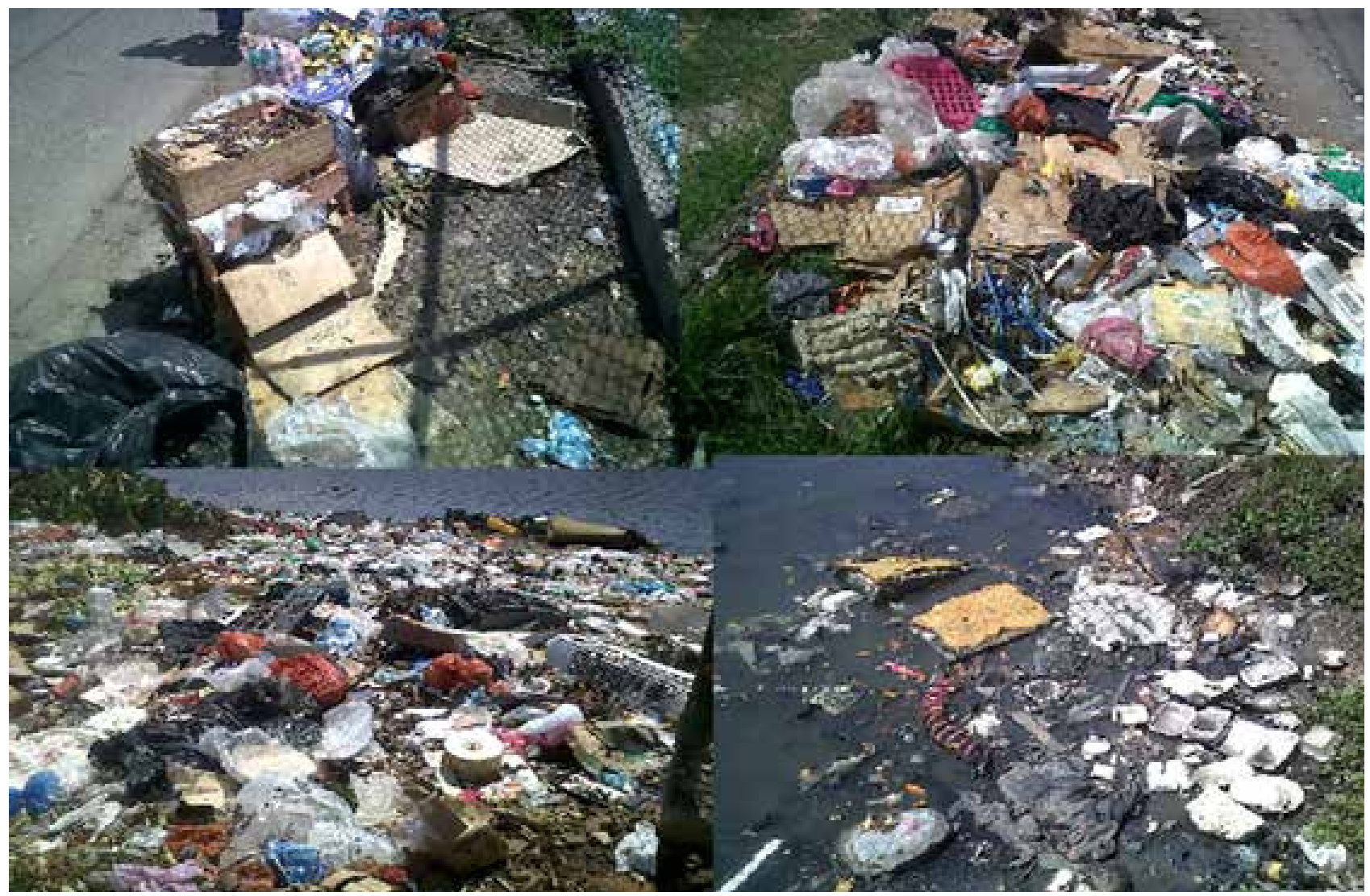

Fig. 1. Ejemplo de inadecuada disposición final de residuos sólidos en las inmediaciones de la Ciénaga de Las Quintas, sector Bazurto, Cartagena.

2011). A pesar de ello, Colombia presenta serias desigualdades a lo largo de su territorio, como por ejemplo, la situación del departamento de Bolívar, donde en 2008 apenas el 45\% de la población recibió un servicio adecuado de recolección de residuos. Se estima que el departamento dispone unas 150 Ton/día de residuos de forma descontrolada, por lo general en los cuerpos de agua y suelos abandonados. En el caso del sector rural, el departamento de Bolívar tiene una cobertura de recolección de residuos de apenas 0,5\%, contra la cobertura nacional promedio de 1,9\% (DAP, 2011).

En países en desarrollo como Colombia, los residuos domésticos están compuestos hasta 2/3 de material orgánico, con densidades de 300 a $600 \mathrm{Kg} / \mathrm{m}^{3}$ (UN-HABITAT, 2010a). Los restaurantes callejeros, vendedores de jugos de frutas y mercados públicos generan grandes cantidades de residuos de alimentos que en muchos casos reciben un servicio de recolección pobre o inadecuado, el cual, combinado con los malos hábitos culturales de la población, genera la acumulación de residuos en las calles, muchas veces junto a cuerpos de agua. Los residuos dispuestos en las calles suelen bloquear los sistemas de alcantarillado causando inundaciones, estancamiento de agua, cría de mosquitos y otros vectores de enfermedades y la contaminación de los cuerpos de agua que normalmente utiliza la población para abastecerse para consumo, cocción y limpieza. Específicamente en Colombia, las altas temperaturas sumadas a las condiciones de humedad ambiente, aceleran el proceso de degradación de los residuos, aumentando la generación de lixiviados y los efectos de los mismos sobre los ecosistemas (UN-HABITAT, 2010b). Un ejemplo de esta situación se observa en los alrededores de la Ciénaga de Las Quintas, la cual limita con el Mercado de Bazurto en Cartagena de Indias, donde los residuos sólidos (en su mayoría restos de alimentos) se acumulan en las calles y junto a la ciénaga, tal como se observa en la Figura 1, presentando procesos de degradación in situ y generando olores ofensivos y gases, entre ellos el metano, que se liberan a la atmósfera.

Los residuos orgánicos domésticos tienen un potencial de generación de metano de 0,1 a 0,93 $\mathrm{m}^{3} / \mathrm{Kg}$ de materia seca (Surendra, 2014), valor que depende de la composición de los mismos. Teniendo en cuenta que una familia de 3-4 miembros consume alrededor de $0,5 \mathrm{~m}^{3}$ de gas para cocción al día, se necesitaría un máximo de $5 \mathrm{Kg}$ de materia seca/día para autoabastecerse, cantidad que se reduce si se combinan con residuos de elevado potencial o mediante la codigestión. Estas cantidades no son fáciles de obtener a nivel doméstico en una ciudad; sin embargo, a nivel rural, si se suman los residuos procedentes de la preparación de alimentos con los restos de las actividades agrícolaganaderas, es posible alcanzar niveles de producción constantes que permitan el autoabastecimiento. 


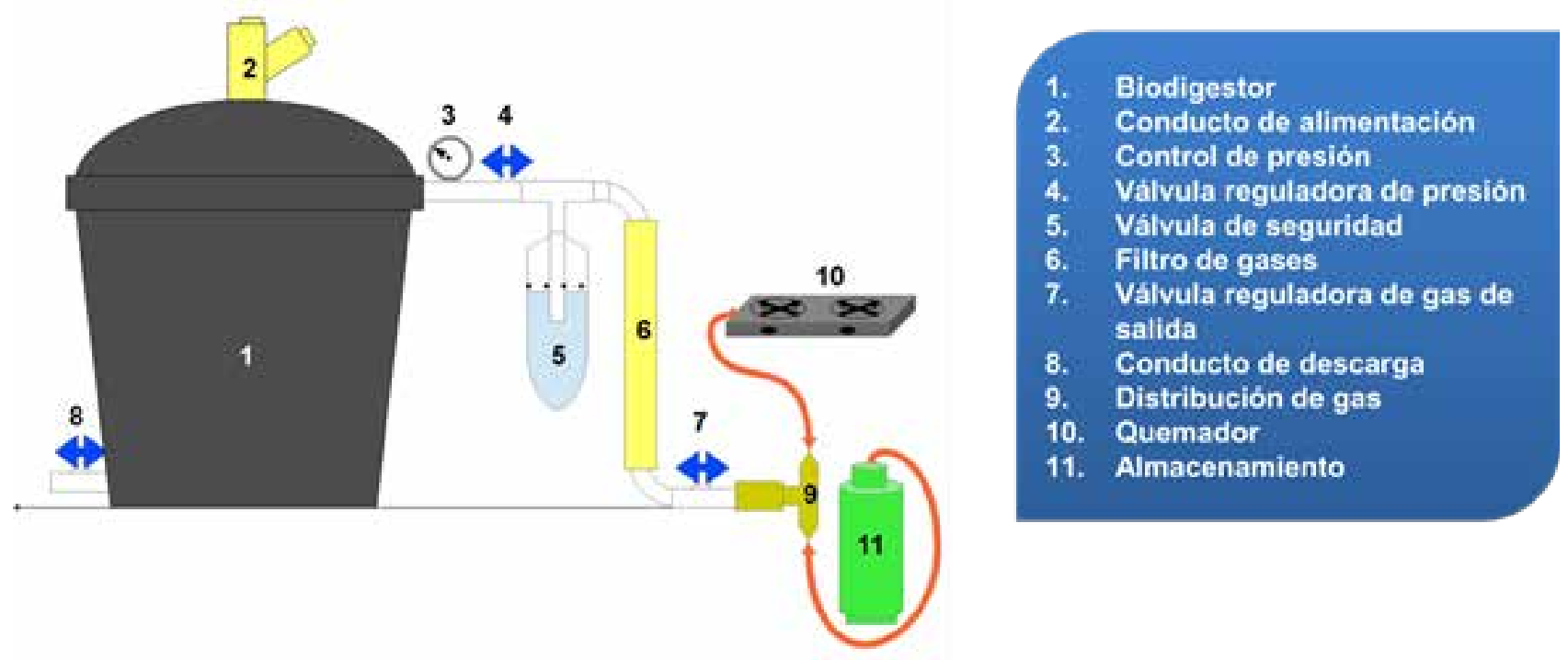

Fig. 2. Esquema de prototipo de biodigestor para autoabastecimiento

En la Figura 2, se presenta un esquema de biodigestor propuestoparaaplicaciones domésticas,cuyofuncionamiento ha sido evaluado con residuos de estiércol animal y restos de alimentos (Acosta, 2014). A diferencia de los modelos de biodigestores utilizados en China e India, de tipo domo fijo o flotante, los cuales requieren obra civil, espacio fijo disponible para su instalación y un coste de inversión inicial a veces elevado, el prototipo propuesto puede montarse y trasladarse fácilmente de acuerdo a la necesidad, lo que permitiría su disponibilidad comercial y fácil distribución a los usuarios finales. Los dispositivo semienterrados generan a menudo a la población una sensación de inseguridad en el suministro y operación (Zhou, 2011), y es por ello que el modelo propuesto se ha pensado para una fácil operación y mantenimiento (Acosta, 2014). El desarrollo de este tipo de proyectos en las $Z N I$ de Colombia facilitaría el acceso a esta tecnología.

Finalmente, en los últimos años se ha observado en Colombia el creciente interés de las autoridades por el fomento de las FNCE y de estrategias de aprovechamiento de residuos. Un ejemplo de ellos es la reciente Ley 1715 (2014), que en su artículo 18 (Energía de Residuos) indica que "Será considerado como Fuentes No Convencionales de Energía Renovable (FNCER) el contenido energético tanto de la fracción biodegradable, como de la fracción de combustible de los residuos de biomasa." Con la implementación de dicha ley se van a promover y a estimular en Colombia todas las fuentes de energía no convencionales, tales como las procedentes de la biomasa, en particular el biogás. Además, la nueva ley contribuirá a las soluciones energéticas para las zonas rurales y otras zonas actualmente aisladas del sistema interconectado nacional.

Por otro lado, el Decreto 2981 de 2013, en su Artículo 82 (Propósitos del aprovechamiento) propone: "Racionalizar el uso y consumo de las materias primas provenientes de los recursos naturales. Recuperar valores económicos y energéticos que hayan sido utilizados en los diferentes procesos productivos. Aumentar la vida útil de los rellenos sanitarios al reducir la cantidad de residuos a disponer finalmente en forma adecuada. Reducir el caudal y la carga contaminante de lixiviados en el relleno sanitario, especialmente cuando se aprovechan residuos orgánicos". De este modo, la aplicación del mencionado decreto fomenta el uso energético de los residuos tales como los de alto contenido orgánico y la reducción de la cantidad que se dispone en rellenos sanitarios. Ambas metas pueden alcanzarse con la aplicación de tecnologías de biogás.

\section{CONCLUSIONES}

Colombia genera grandes cantidades de residuos urbanos, principalmente de tipo orgánico. En los últimos años se ha estado fomentando el uso de las $F N C E$, especialmente para zonas no interconectadas. Colombia dispone de una gran variedad de materias primas para producción de biogás. Existen hasta el momento escasos proyectos de producción de biogás, por ejemplo, en el sector del cultivo de Palma, pero a nivel doméstico, especialmente en el sector rural, la aplicación es aún escasa. La elevada disponibilidad de residuos de tipo orgánico, las condiciones climáticas y la necesidad de cubrir las necesidades energéticas de la población de las $Z N I$ de una forma limpia y económica son factores que combinados deberían potenciar el uso de la tecnología de biogás a nivel doméstico.

AGRADECIMIENTOS Los autores agradecen el apoyo brindado por la Fundación Universitaria Tecnológico Comfenalco por la financiación del proyecto, en particular a los programas ambientales, al grupo de investigaciones GIA $y$ al Semillero IGA. 


\section{REFERENCIAS}

Acosta, M.E \& Pasqualino, J.C. (2014). Aprovechamiento integral de residuos sólidos orgánicos mediante generación de biogás y fertilizante. Encuentro Institucional de Semilleros de Investigación del Tecnológico Comfenalco - ESITC 2014.

Arrieta, F.R.P., Teixeira, F.N., Yáñez, E., Lora, E. \& Castillo, E. (2007). Cogeneration potential in the Columbian palm oil industry: Three case studies. Biomass and Bioenergy, 31(7),503-511.

Biotec. (2013). Número especial: Biodigestores para extractoras de aceite de palma. Newsletter Biotec External Bulletin, April 2013.

Cendales Ladino, E.D. (2011). Producción de biogás mediante la codigestión anaeróbica de la mezcla de residuos cítricos y estiércol bovino para su utilización como fuente de energía renovable. (Tesis de maestría). Universidad Nacional de Colombia, Facultad de Ingeniería, Bogotá, Colombia. Recuperado de http://www.bdigital.unal.edu. $\mathrm{co} / 4100 /$

Conil, P. \& Kervyn B. (2009). Biogas con Efluentes de Palma: desde los Primeros Biodigestores de los Años 80 hasta los MDL (Mecanismos de Desarrollo Limpio) de los Años 2000. Proceedings of the Palm Oil International Palm Oil Congress - PIPOC 2009. Recuperado de http://200.29.232.126/ wordpress/wp-content/uploads/2013/02/42-PC27-C77-_ PIPOC-publicacion-espanol_-Sept-2-09.pdf

Decreto 2928, Por el cual se reglamenta la prestación del servicio público de aseo (2013). República de Colombia. Ministerio de Vivienda, Ciudad y Territorio.

Departamento Nacional de Planeación, Colombia (2011). Plan Nacional de Desarrollo 2010-2014: Prosperidad para todos, Tomo II. Bogotá: Colombia. Imprenta Nacional de Colombia. ISBN: 978-958-8340-70-8. Recuperado de https://sinergia.dnp.gov.co/SISMEG/Archivos/PND20102014\%20Tomo\%20II\%20CD.pdf

Departamento Administrativo de Planeación, Bolívar, Colombia (2012). Plan de Desarrollo Departamento de Bolívar 2012-2015: Bolívar Ganador. Cartagena, Bolívar: Colombia. Recuperado de http://www.bolivar.gov.co/ index.php?option=com_rokdownloads\&view=file\&task =download\&id=1925\%3Adocumento-tecnico-plan-dedesarrollo-bolivar-ganador-2012-2015\&Itemid=316

Ferrer, I., Garfí, M., Uggetti, E., Ferrer-Martí, L., Calderon, A. \& Velo, E. (2011). Biogas production in low-cost household digesters at the Peruvian Andes. Biomass and Bioenergy, 35(5),1668-1674.
Flotats, X., Palatsi, J., Fernández, B., Colomer, M. À. \& Illa, J. (2010). Identifying anaerobic digestion models using simultaneous batch experiments. Environmental Engineering and Management journal, 9(3), 313-318.

Guardado Chacón, J.A. (2007). Diseño y construcción de plantas de biogás sencillas. Editorial CUBASOLAR

Instituto de Investigación de Recursos Biológicos Alexander von Humboldt $(\mathrm{IAvH})$, Instituto de Hidrología, Meteorología y Estudios Ambientales (IDEAM), Instituto de Investigaciones Ambientales del Pacífico (IIAP), Instituto de Investigaciones Marinas y Costeras (INVEMAR), Instituto Amazónico de Investigaciones Científicas (SINCHI) (2011). Informe del Estado del Medio Ambiente y de los Recursos Naturales Renovables 2010. Bogotá: Colombia. Imprenta Nacional de Colombia. ISBN: 978958-8067-35-3. Recuperado de http://documentacion. ideam.gov.co/openbiblio/Bvirtual/022166/022166.htm.

Ley 1715 , por medio de la cual se regula la integración de las energías renovables no convencionales al sistema energético nacional (2014). República de Colombia. Congreso de Colombia.

Ministerio de Minas y Energía, Colombia (2010). Programa de Uso Racional y Eficiente de Energía y Fuentes No Convencionales - PROURE. Plan de acción 2010-2015 con visión al 2025. Bogotá: Colombia. Recuperado de http://www.minminas.gov.co/minminas/downloads/ UserFiles/File/ENERGIA/URE/Informe_Final_Consultoria_ Plan_de_accion_Proure.pdf

Mwirigi, J., Balana, B., Mugisha, J., Walekhwa, P., Melamu, R., Nakami, S. \& Makenzi, P. (2014). Socio-economic hurdles to widespread adoption of small-scale biogas digesters in Sub-Saharan Africa: A review. Biomass and Bioenergy, In press, doi: 10.1016/j.biombioe.2014.02.018.

Orskov, B., Yongabi, K., Subedi, M. \& Smith, J. (2014). Overview of holistic application of biogas for small scale farmers in Sub-Saharan Africa. Biomass and Bioenergy, In press, doi: 10.1016/j.biombioe.2014.02.028.

Pasqualino, J., Meneses, M., Abella, M. \& Castells, F. (2009). LCA as a Decision Support Tool for the Environmental Improvement of the Operation of a Municipal Wastewater Treatment Plant. Environmental Science and Technology, 43, 3300-3307. doi: 10.1021/es802056r

Pérez, I., Garfí, M., Cadena, E. \& Ferrer, I. (2014). Technical, economic and environmental assessment of household biogas digesters for rural communities. Renewable Energy, 62,313-318. 
Pinzón A., M.C. (2004). Biblioteca ilustrada del campo: Abonos orgánicos biodigestores-biopreparados-humussuelos. Editorial Enlace Cultural.

Raha, D., Mahanta, P.\& Clarke, M. (2014). The implementation of decentralized biogas plants in Assam, NE India: The impact and effectiveness of the National Biogas and Manure Management Programme. Energy Policy, 68, 80-91.

Song, Z., Zhang, Ch., Yang, G., Feng, Y., Ren, G. \& Han, X. (2014). Comparison of biogas development from households and medium and large-scale biogas plants in rural China. Renewable and Sustainable Energy Reviews, 33, 204-213.

Subedi, M., Matthews, R., Pogson, M., Abegaz, A., Balana, A., Oyesiku-Blakemore, J. \& Smith, J. (2014). Can biogas digesters help to reduce deforestation in Africa? Biomass and Bioenergy, In press, DOl: 10.1016/j.biombioe.2014.02.029.

Surendra, K.C., Takara, D., Hashimoto, A.G. \& Khanal, S.K. (2014). Biogas as a sustainable energy source for developing countries: Opportunities and challenges. Renewable and Sustainable Energy Reviews, 31, 846-859. DOl: 10.1016/j.rser.2013.12.015

United Nations Human Settlements Programme (UNHABITAT) 2010a. Collection of Municipal Solid Waste in Developing Countries. Malta. Gutenberg Press. ISBN 97892-1-132254-5. Recuperado de http://mirror.unhabitat. org/pmss/getElectronic Version.aspx?nr=3072\&alt=1

United Nations Human Settlements Programme (UNHABITAT) 2010b. Solid Waste Management in the World Cities. State of Water and Sanitation in the World Cities. Malta. Gutenberg Press. ISBN 978-184971-169-2. Recuperado de mirror.unhabitat.org/pmss/ getElectronic Version.aspx?nr=2918\&alt=1

Walekhwa, P.N., Lars, D. \& Mugisha, J. (2014). Economic viability of biogas energy production from family-sized digesters in Uganda. Biomass and Bioenergy, In press, doi: 10.1016/j.biombioe.2014.03.008.

Zhou, X.Z., Ou, S.L., \& Huang C.I. (2011). Problems and Solutions Based on Comprehensive Utilization of Biogas. Energy Procedia, 5, 42-47. 